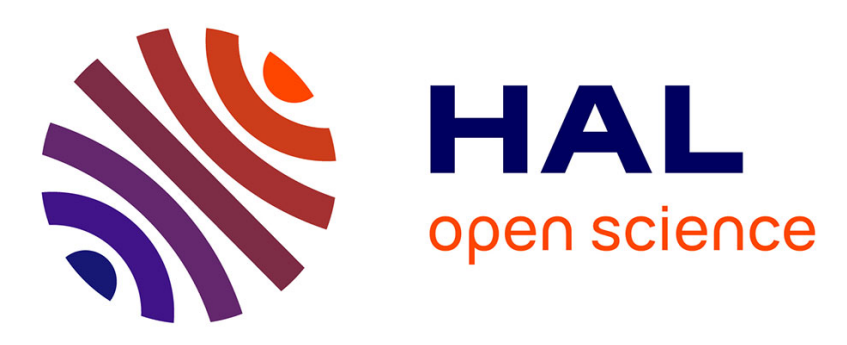

\title{
Scapular kinematics during scaption in competitive swimmers
}

\author{
Yoann Blache, Benoit Gillet, J. Selin, V. Sevrez, I. Rogowski
}

\section{To cite this version:}

Yoann Blache, Benoit Gillet, J. Selin, V. Sevrez, I. Rogowski. Scapular kinematics during scaption in competitive swimmers. European Journal of Sport Science, 2018, 18 (5), pp.659 - 666. 10.1080/17461391.2018.1449893 . hal-01926641

\section{HAL Id: hal-01926641 \\ https://univ-lyon1.hal.science/hal-01926641}

Submitted on 19 Nov 2018

HAL is a multi-disciplinary open access archive for the deposit and dissemination of scientific research documents, whether they are published or not. The documents may come from teaching and research institutions in France or abroad, or from public or private research centers.
L'archive ouverte pluridisciplinaire $\mathbf{H A L}$, est destinée au dépôt et à la diffusion de documents scientifiques de niveau recherche, publiés ou non, émanant des établissements d'enseignement et de recherche français ou étrangers, des laboratoires publics ou privés. 


\title{
Scapular kinematics during scaption in competitive swimmers
}

\author{
Blache $\mathrm{Y},{ }^{1}$ Gillet B, ${ }^{1,2}$ Selin J, ${ }^{1}$ Sevrez V,${ }^{3}$ Rogowski I ${ }^{1}$
}

${ }^{1}$ Univ Lyon, Université Claude Bernard Lyon 1 ; Laboratoire Interuniversitaire de Biologie de la Motricité - EA 7424, UFRSTAPS, 27-29 boulevard du 11 novembre 1918, 69622 Villeurbanne Cedex, France ; +33472432848; isabelle.rogowski@ univ-lyon1.fr

${ }^{2}$ Laboratoire de Simulation et de Modélisation du Mouvement (S2M), Département de Kinésiologie, Université de Montréal, Laval, QC, Canada

${ }^{3}$ Aix-Marseille Université, Institut des Sciences du Mouvement Etienne-Jules Marey, Marseille, France

\begin{abstract}
This study aimed 1) to describe and compare scapular kinematics between three groups of swimmers of different levels and a group of non-swimmers, and 2) to assess whether swimming practice alters the asymmetries in scapular kinematics between the dominant and non-dominant sides, both during unilateral arm raising and lowering in the scapular plane. Scapular kinematics were assessed bilaterally during arm raising and lowering in the scapular plane using an electromagnetic system in 42 healthy males, which were split into four groups: control $(n=11)$, adolescent elite swimmers ( $n=11)$, adult elite swimmers $(n=10)$, and club-level adult swimmers $(n=10)$. One-Way ANOVA SPM(t) on two repeated measures showed that the three groups of swimmers had more protracted shoulder between $30^{\circ}$ and $90^{\circ}$ of arm raising and lowering $(\mathrm{p}<0.001)$. The three groups of swimmers presented no bilateral difference in scapular upward rotation, while the dominant scapula was more upwardly rotated than the non-dominant one between $74^{\circ}$ and $104^{\circ}$ of arm elevation in the control group $(\mathrm{p}<0.001)$. The scapula of adult elite swimmers was more internally rotated between $67^{\circ}$ and $116^{\circ}$ of humeral elevation during arm raising, and between $81^{\circ}$ and $54^{\circ}$ during arm lowering in comparison to the other swimming and control groups $(\mathrm{p} \leq 0.02)$, who presented similar scapular positioning in internal
\end{abstract}


rotation. In conclusion, the findings of the study pointed out that swimming practice generated protracted shoulders and removed bilateral differences in scapular upward rotation during scaption, while accumulation of swimming practice at elite level enlarged scapular internal rotation.

Key words: Skill-level; Swimming; Shoulder complex adaptations; Scapular Dyskinesis; Shoulder

\section{Introduction}

Swimming at competitive level demands a training volume ranged between $9 \mathrm{~km} / \mathrm{week}$ for club-level swimmers and $110 \mathrm{~km} /$ week or more for international level swimmers (Sein, Walton et al. 2010). The corresponding number of arm revolutions is estimated between 3000 and 44000 per week (Bradley, Bowmaker et al. 2016) which cumulate during the 40 weeks (Hibberd, Oyama et al. 2012) of each swimmer's career season. Repeated arm revolutions involve a large amount of musculoskeletal loads onto the shoulder complex, which is known to generate alteration in shoulder kinematics (Hibberd, Oyama et al. 2012, Hibberd, Laudner et al. 2016), although these alterations in relation with skill-level of the swimmers are not yet well described.

During free-style stroke, both arms act alternatively but in the same fashion (Bak and Magnusson 1997). Depending on the studies (Pink, Perry et al. 1991, Chollet, Chalies et al. 2000, Yanai and Hay 2000), each arm revolution may be divided into three successive phases. The catch phase occurs from the hand entry in the water, during which the shoulder is elevated to reach forward maximally (Yanai and Hay 2000). Then the pull phase demands powerful humeral adduction, internal rotation and extension (Pink, Perry et al. 1991, Du and Yanai 2016). 
Finally, the recovery phase is achieved by a humeral abduction and external rotation to place the hand forward for the following arm entry (Yanai and Hay 2000). In consequence, the bilateral nature of swimming may involve similar adaptations in the dominant and nondominant shoulders, (Madsen, Bak et al. (2011), leading to annihilate the bilateral shoulder asymmetry observed in sedentary population (Matsuki 2011, Morais 2013).

Acute response to excessive repeated arm revolutions involves an alteration in scapular kinematics in swimmers. Madsen, Bak et al. (2011) observed that $82 \%$ of their adolescent swimmers presented scapular dyskinesia during arm elevation in the scapular plane after 25 minutes of swimming training. Prevalence of such scapular dyskinesia increased through a training session (Madsen, Bak et al. 2011). Alterations in scapular kinematics, such as increased internal rotation, protraction and elevation has been also observed after a 6-week swimming training period in young adults (Hibberd, Oyama et al. 2012). Besides, long-term swimming is known to enlarge and tighten the anterior shoulder musculature causing postural adaptations, such as forward head and rounded shoulders in adolescents (Laudner, Wenig et al. 2015, Hibberd, Laudner et al. 2016). However, all these studies have been performed on adolescents and young adults (between 16.5 and 19 years old on average), while the amount of shoulder adaptations due to repeated arm revolutions may depend on the mileage per week, the number of years of practice (partially related to the age of the swimmer) and the level of practice (Gordon 2004).

Finally, shoulder postural adaptations observed in swimmers may affect the scapular positioning during arm motion. Indeed, previous study involving a healthy adult population has shown that increased forward posture, demands more shoulder elevation, less upward rotation, less posterior tilt and more internal rotation to elevate the arm in the scapular plane (Kebaetse, McClure et al. 1999). As such scapular positioning has been associated with various shoulder 
injuries (Kibler, Ludewig et al. 2013), it is important to define the normal scapular kinematics in healthy swimmer athletes in order to contribute to shoulder injury prevention.

The objectives of this study were 1) to describe and compare scapular kinematics between three groups of swimmers of different levels and a group of non-swimmers, and 2) to assess whether swimming practice alters the asymmetries in scapular kinematics between the dominant and non-dominant sides, both during unilateral arm raising and lowering in the scapular plane. It was hypothesized that the swimmers' shoulder adapts to the swimming practice by increasing shoulder elevation and protraction as well as by increasing scapular internal rotation and anterior tilt while decreasing scapular upward rotation and eliminating handedness effect.

\section{Methods}

\section{Participants}

Forty-two healthy male adults participated to this study. They signed an informed consent document, which was approved by the Ethical Committee "Sud-Est II" (IRB 00009118). None of the participants declared any shoulder and upper limb symptoms and injuries during the 12 months prior to the experimentation. Then, the participants were split into four groups (Table 1). The first group (G0_Cont) was composed of 11 males that did not practice swimming or any sports involving the shoulder and upper limbs, and was used as the control group. The second group (G1_AdoElit) included 11 adolescent elite swimmers. The third group (G2_AduElit) included 10 adult elite swimmers. The last group (G3_AduClub) was composed of 10 club-level adult swimmers. The practice level was determined by the French federation quotation, which attributes between 1 and 1500 points to the swimmer based on his/her best race during the year preceding the study (French Federation of Natation, 2016, https://ffn.extranat.fr/html/ressources/nat/table_cotation_2017.pdf). 
Table 1: Characteristics of the four groups.

\begin{tabular}{lcccc} 
& G0_Cont & G1_AdoElit & G2_AduElit & G3_AduClub \\
\hline $\mathrm{n}$ & 11 & 11 & 10 & 10 \\
Age (years) & $23.2 \pm 1.7$ & $17 \pm 1.0^{\#}$ & $21.9 \pm 2.2$ & $20.8 \pm 4.4$ \\
Height (m) & $1.73 \pm 0.10$ & $1.86 \pm 0.07^{\# !}$ & $1.84 \pm 0.06^{\# !}$ & $1.76 \pm 0.07$ \\
Mass (kg) & $68.2 \pm 13.5$ & $71.1 \pm 7.1$ & $76.9 \pm 8.7$ & $69.2 \pm 11.1$ \\
Quotation (points) & $0.0 \pm 0.0$ & $1192 \pm 59^{\# !}$ & $1248 \pm 83^{\# !}$ & $1009 \pm 138^{\#}$ \\
Swimming & $0.0 \pm 0.0$ & $10.5 \pm 2.3^{\#}$ & $13.9 \pm 5.1^{\# \dagger}$ & $13.7 \pm 4.7^{\# \dagger}$ \\
Experience (years) & & $17.9 \pm 3.3^{\ddagger}$ & $15.5 \pm 4.6^{\ddagger}$ & $8.1 \pm 4.4$ \\
Training/week (h) & NA & & \\
\hline
\end{tabular}

\# different from the control group (G0_Cont) at $\mathrm{p}<0.05$

$\$$ different from the adult club-level group (G3_AduClub) at $\mathrm{p}<0.05$

${ }^{\dagger}$ different from the adolescent elite group (G1_AdoElit) at $\mathrm{p}<0.05$

\section{Instrumentation and data collection}

To perform the kinematic analysis, an electromagnetic device, which has already been tested to assess shoulder kinematics (El Habachi, Duprey et al. 2015, Gillet, Berger-Vachon et al. 2015, Seitz, McClelland et al. 2015), was used (100Hz; Trackstar, Ascension Technology Corporation, Chicago, Ill). It was composed of five sensors located on incisura jugularis, flat portion of the right and left acromion angles, and distal portion of the right and left humerus. In order to calibrate the kinematic model and define segments reference system, a sixth sensor was taped to a stylus used to digitalize bony landmarks (T8, processus xiphoideus, $\mathrm{C}$, incisura jugularis, and for both sides, scapular angulus acromialis, trigonum scapulae, angulus inferior, humeral medial and lateral epicondyle).

In a previous experiment (non-published data), we observed that our electromagnetic device used in the same conditions than the present study ensured fair to good intersession relative reliability $(\operatorname{ICC}[2,1]=0.81 \pm 0.15)$ and an absolute reliability $(\mathrm{SEM})$ of $4.2 \pm 1.4^{\circ}$. In addition, a 
previous study reported errors of scapular kinematics during arm elevation included between 5 and $9^{\circ}$ in comparison to the palpation method (El Habachi, Duprey et al. 2015).

\section{Procedure}

Prior to the tests, the participants kept a standing upright posture for further calibration. Then they performed four arm circumductions to determine joint center of rotation. Finally, they performed several arms elevation with a maximal amplitude to be familiarized with the procedure and the instruments. The tests consisted in raising and lowering the arm in the scapular plane with the thumb pointed in the upward direction. The participants had to keep the elbow straight throughout the movement. Two cycles of unilateral arm raising/lowering were performed with the dominant (i.e. writing hand) and non-dominant arms in a controlled random order. In order to ensure that the movements were performed in the scapular plane, a goniometer was used to set the plane of elevation ( $30^{\circ}$ anterior to the frontal plane). Thereafter, a vertical pole was located in scapular plane of elevation and guided the participant throughout the movement. Finally, a cycle duration lasted four seconds (two seconds for raising and lowering) (Ludewig and Cook 2000).

\section{Kinematics}

Raw data were filtered using a low-pass $4^{\text {th }}$ order Butterworth filter with a cut-off frequency of $10 \mathrm{~Hz}$. The local coordinate systems were defined in line with the recommendations of the International Society of Biomechanics (Wu, van der Helm et al. 2005). Firstly, clavicular orientation relative to the thorax were described by two rotations, namely protraction/retraction and elevation/depression (McClure, Michener et al. 2001, Myers, Laudner et al. 2005) (Figure1). To that aim, the vector from the incisura jugularis (IJ) and Acromion Angle (AA) points was used. Clavicular elevation/depression corresponded the angle between the vector 
$\overrightarrow{I J-A A}$ and the transversal plane of the thoracs that bisects IJ point. For the clavicular protraction/retraction, the vector $\overrightarrow{I J-A A}$ was projected onto the transverse plane bisecting IJ. Then, clavicular protraction/retraction was the angle between the projected vector and the frontal plane of the thorax that bisects IJ (McClure, Michener et al. 2001, Myers, Laudner et al. 2005). Secondly, scapular orientation with respect to the thorax were computed with Euler angle decompositions (Figure 1). Scapulothoracic rotations were defined as internal/external rotation, upward/downward rotation, and anterior/posterior tilt, using YXZ rotation sequence. Humerothoracic rotations were determined as the plane of elevation, elevation, and rotation using YXY rotation sequence. In order to ensure a sufficient accuracy of the assessment of scapular kinematics, arm elevations were analyzed between 30 and $120^{\circ}$ of humerothoracic elevation (Karduna, McClure et al. 2001, Senk and Chèze 2010). The mean of the two trials were computed for the analysis.
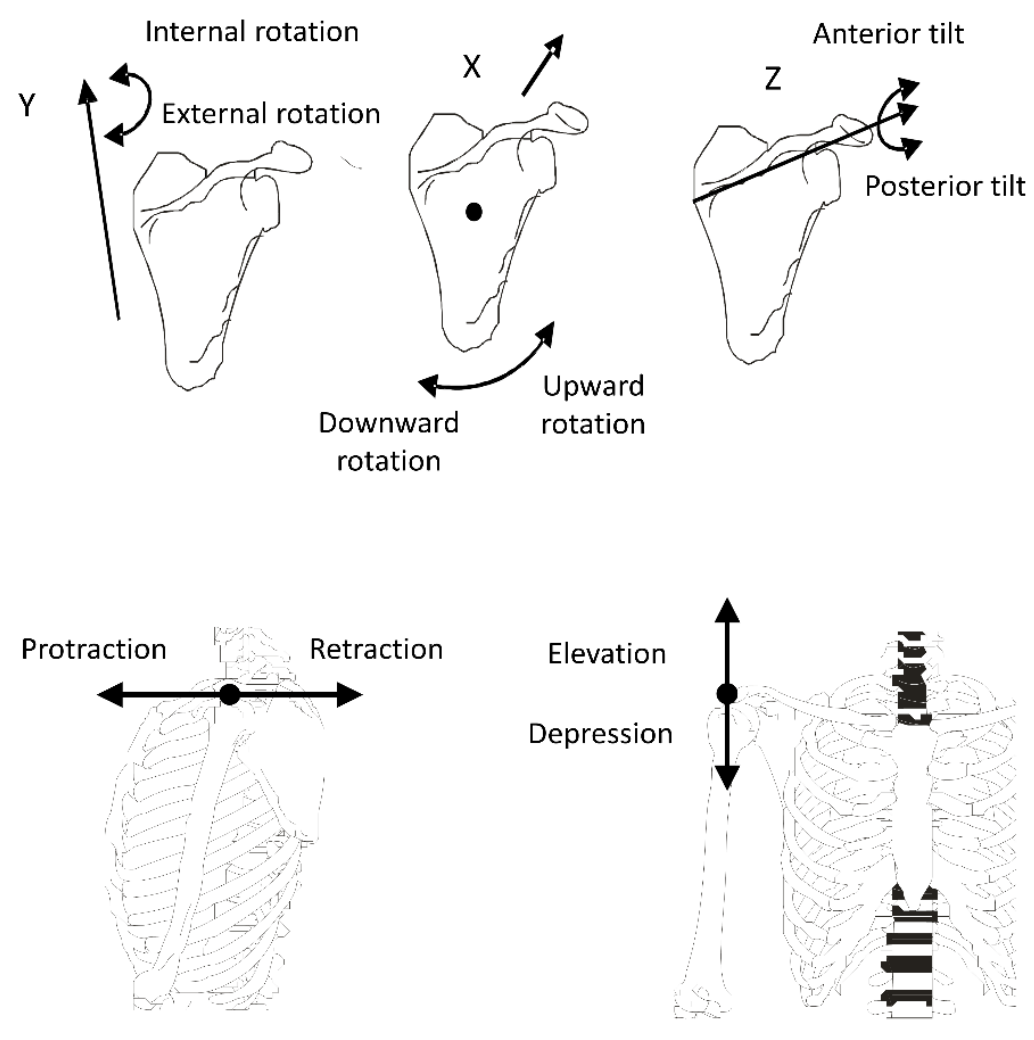

Figure 1- Clavicular and scapular orientations 


\section{Statistics}

All statistical tests were performed with the open-source toolbox SPM-1D (OTodd Pataky 2014, version M0.1) that performs Statistical Parametric Mapping on 1-dimensional timeseries. Statistical Parametric Mapping is a statistical technique that was developed in the field of neuro-imaging, and was adapted by Pataky (2008) to allow statistical comparisons for onedimensional series in biomechanics field. This technique is used to assess for differences between sets of curves. Inferential statistics in Statistical Parametric Mapping technique compare the observed cluster to a random cluster to determine significant difference in the cluster widths, i.e. difference in clavicular and scapular positioning for a given range of arm elevation. The effect of the group (G0_Cont, G1_AdoElit, G2_AduElit and G3_AduClub) and the laterality (dominant and non-dominant sides) on the scapulothoracic rotations was assessed by a One-Way ANOVA SPM(t) on two repeated measures. When an interaction effect or a main effect of the group was observed, post-hoc tests were applied. The level of significance was set at $\mathrm{p}<0.05$.

\section{Results}

In all groups, the shoulder elevated during arm raising and depressed during arm lowering (see supplementary file S1). Statistical analysis revealed no significant effect of interaction, or group or laterality for shoulder elevation/depression either during arm raising or lowering.

The shoulder retracted during arm raising and protracted during arm lowering (Figure 2 and supplementary file S2). ANOVA revealed only a significant effect of the group for the shoulder protraction/retraction between 30 and $120^{\circ}$ of humeral elevation during the arm raising and lowering. Post-hoc tests showed that the shoulders were more retracted in G0_Cont in comparison to G1_AdoElit, G2_AduElit and G3_AduClub $(\mathrm{p}<0.001, \mathrm{p}=0.03$ and $\mathrm{p}<0.001$, 
respectively). Shoulders were more retracted for G2_AduElit when compared to G1_AdoElit and G3_AduClub ( $\mathrm{p}=0.004, \mathrm{p}=0.04$, respectively), while similar shoulder retraction was observed for the shoulders of G1_AdoElit and G3_AduClub.

For all groups, the scapula internally rotated during arm raising and externally rotated during arm lowering (Supplementary file S3). ANOVA revealed only a significant effect of the group between $67^{\circ}$ and $116^{\circ}$ of humeral elevation during arm raising, and between $81^{\circ}$ and $54^{\circ}$ during arm lowering (Figure 3). The scapular positioning in internal rotation was similar in G0_Cont, G1_AdoElit and G3_AduClub, while G2_AduElit presented increased scapular internal rotation $(\mathrm{p}=0.005, \mathrm{p}=0.02$ and $\mathrm{p}=0.007$, respectively).

In all groups, the scapula upwardly rotated during arm raising and downwardly rotated during arm lowering (Figure 4). ANOVA revealed a significant effect of the interaction group $*$ laterality between $74^{\circ}$ and $104^{\circ}$ of humeral elevation during arm raising. Post hoc tests showed a significant side-to-side difference in G0_Cont $(\mathrm{p}<0.001)$, while no significant sideto-side difference was observed in all the other groups of swimmers. The non-dominant scapular upward rotation was significantly higher in G0_Cont when compared to G2_AduElit $(\mathrm{p}=0.03)$ and G3_AduClub ( $\mathrm{p}=0.01)$, while no difference was observed between the four groups for the dominant side.

In all groups, the scapula posteriorly tilted during arm raising and anteriorly tilted during arm lowering (supplementary file S4). Statistical analysis revealed no significant effect of interaction, or group or laterality for scapular anterior/posterior tilt either during arm raising or lowering. 


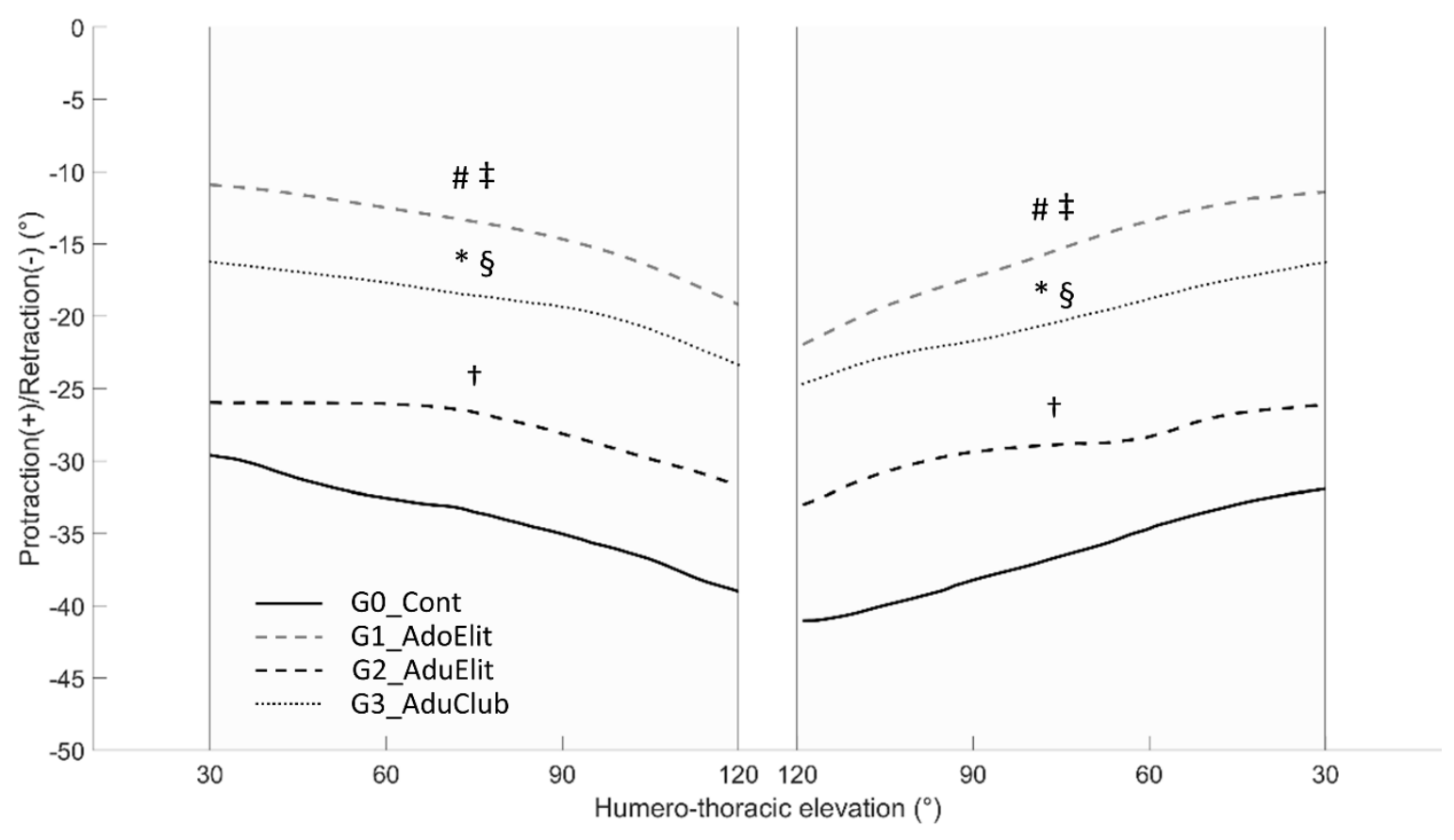

Figure 2- Mean protraction/retraction with respect to the humerothoracic elevation for G0_Cont (black solid line), G1_AdoElit (grey dashed line), G2_AduElit (black dashed line), and G3_AduClub (black dotted line).

\# means a significant difference between G0_Cont and G1_AdoElit at $\mathrm{p}<0.05$ inside the grey shaded zone

$\dagger$ means a significant difference between G0_Cont and G2_AduElit at $\mathrm{p}<0.05$ inside the grey shaded zone

* means a significant difference between G0_Cont and G3_AduClub at p $<0.05$ inside the grey shaded zone

$\S$ means a significant difference between G2_AduElit and G3_AduClub at $\mathrm{p}<0.05$ inside the grey shaded zone

$\$$ means a significant difference between G2_AduElit and G1_AduClub at $\mathrm{p}<0.05$ inside the grey shaded zone 


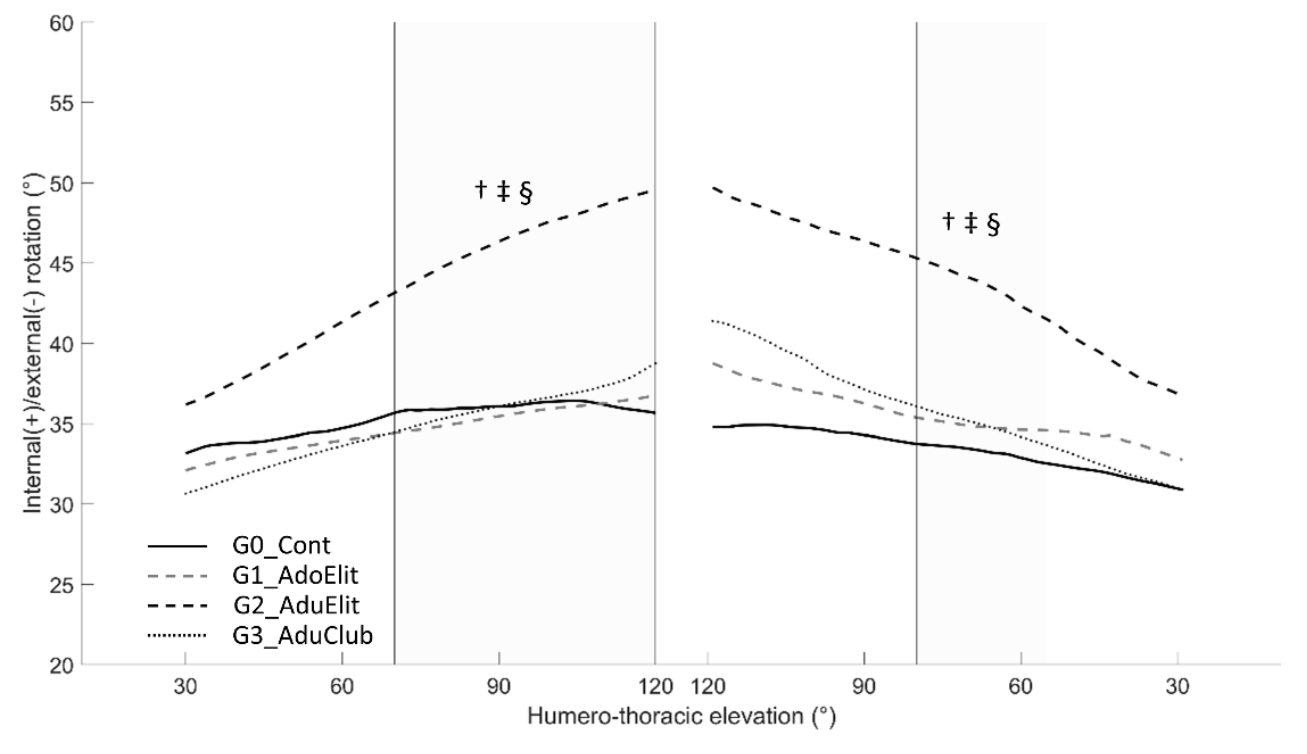

Figure 3: Mean internal/external rotation of the scapula with respect to the humerothoracic elevation for G0_Cont (black solid line), G1_AdoElit (grey dashed line), G2_AduElit (black dashed line), and G3_AduClub (black dotted line).

$\dagger$ means a significant difference between G0_Cont and G2_AduElit at $\mathrm{p}<0.05$ inside the grey shaded zone

\$ means a significant difference between G2_AduElit and G1_AduClub at $p<0.05$ inside the grey shaded zone

$\S$ means a significant difference between G2_AduElit and G3_AduClub at $p<0.05$ inside the grey shaded zone
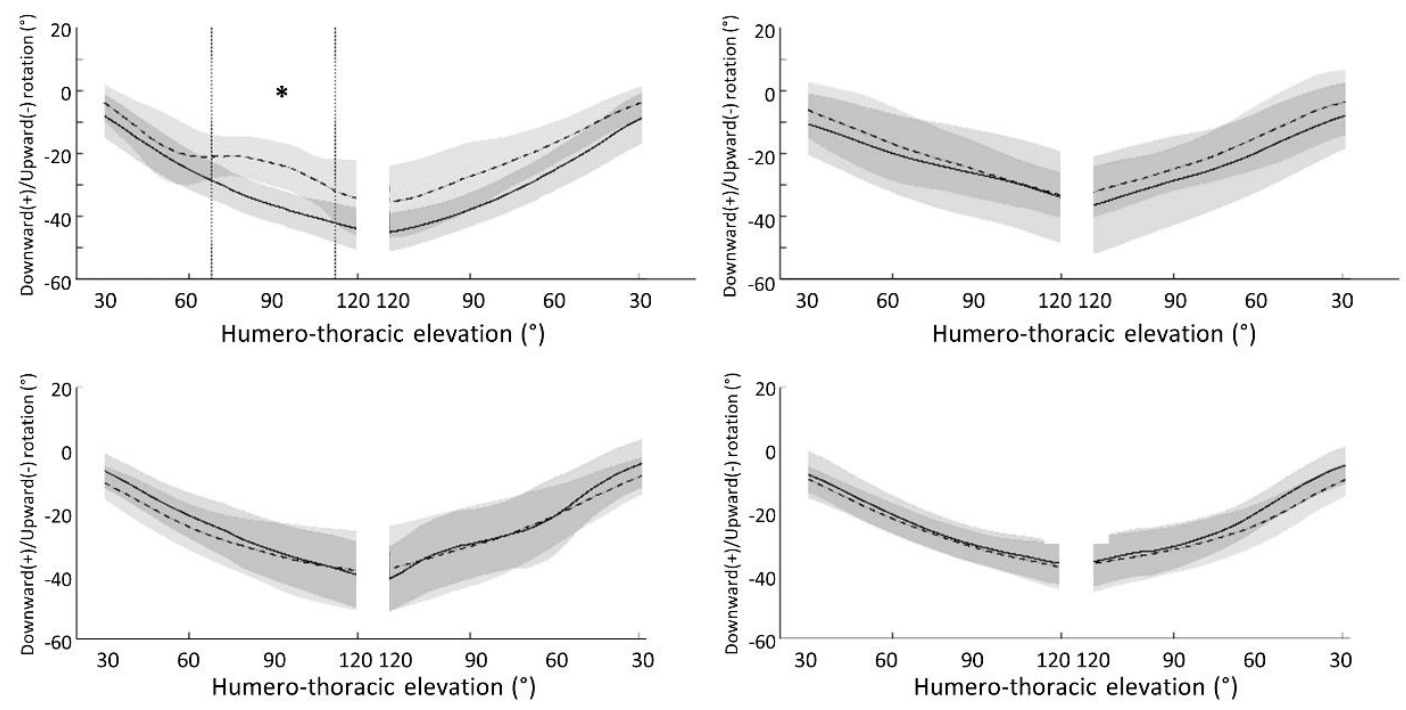

Figure 4: Mean (thick line) \pm 1 standard deviation (shaded zone) downward/upward rotation of the scapula with respect to the humerothoracic elevation for the dominant (blue) and nondominant side (red). G0_Cont: top left, G1_AdoElit: top right, G2_AduElit: bottom left, and 3_AduClub bottom right. * denotes a significant difference between the dominant and nondominant side $(\mathrm{p}<0.05)$. 


\section{Discussion}

The aim of this study was 1) to describe and compare scapular kinematics between three groups of swimmers of different levels and a group of non-swimmers, and 2) to assess whether swimming practice alters the asymmetries in scapular kinematics between the dominant and non-dominant sides, both during unilateral arm raising and lowering in the scapular plane. The main findings confirmed that swimming practice generated an increased clavicular protraction and removed bilateral differences in scapular upward rotation during scaption. The findings of this study also suggested that alterations in scapular internal rotation positioning were related to the swimming practice level.

The achievement of arm motion demands an optimal coordination between the scapula and humerus in order to maintain the congruence of the humeral head into the glenoid cavity (Kibler, Ludewig et al. 2013). For the control group, the clavicular orientation ranged between -37 and $-26^{\circ}$ for the retraction, and 9 and $19^{\circ}$ for the elevation. These values were in the same range of motion than those reported by Myers, Laudner et al. (2005). The scapular orientation in our control group ranged between 35 and $38^{\circ}$ for internal rotation, -8 and $44^{\circ}$ for upward rotation, and -2 and $10^{\circ}$ for anterior tilt. These patterns during scaption in our control group were similar to those reported in the literature for healthy persons (Ludewig, Phadke et al. 2009). We then assumed that the differences in clavicular and scapular kinematics observed between swimmers and controls were related to swimming practice.

Regardless of their specialty, swimmers are known to present a forward posture with a forward head and rounded shoulders (Laudner, Wenig et al. 2015, Hibberd, Laudner et al. 2016). Whatever age or skill-level, our swimmers presented similar adaptations as attested by the increased clavicular protraction in comparison to controls. However, this adaptation was less in adult elite swimmers when compared to adolescent elite or adult club-level swimmers, which may be explained by the strength and conditioning training performed for prevention of 
shoulder injury (Bak 2010). Such training may compensate the forward posture in developing strength of the posterior shoulder musculature (Van de Velde, De Mey et al. 2011), as well as in stretching the anterior shoulder musculature (Gaunt and Maffulli 2012). Contrary to our initial hypotheses, our swimmers did not present increased clavicular elevation when compared to controls. In our study, shoulder kinematics was evaluated up to $120^{\circ}$ of humeral elevation, while the hand entry for freestyle requires an arm elevation of $150^{\circ}$ (Du and Yanai 2016). The lack of clavicular kinematic adaptations until $120^{\circ}$ of humeral elevation may not augur any adaptations for higher humeral elevation.

The forward shoulder posture has been previously associated to an increased scapular internal rotation and anterior tilt, and a decreased scapular upward rotation in a healthy adult population (Kebaetse, McClure et al. 1999), which is quite different from the adaptations in scapular kinematics observed in our swimmers. Indeed, whatever the age and skill-level, our swimmers presented similar dominant scapular upward rotation than controls, while the swimmer's nondominant scapulae were more upwardly rotated when the humerus elevated until $120^{\circ}$ in comparison to controls. Such an adaptation may be beneficial to preserve the clearance of the acromial space at the non-dominant side (Forthomme, Crielaard et al. 2008), and may minimize the risk for subacromial impingement syndrome (Laudner, Wenig et al. 2015). The similar bilateral upward rotation positioning of both the scapulae up to $120^{\circ}$ of humeral elevation observed in our study were in line with previous results assessing scapular upward rotation in static arm elevation at 90 and $140^{\circ}$ in a large group of adolescent swimmers (McLaine, Ginn et al. In Press). Our findings revealed that the elimination of the side-to-side differences in scapular upward rotation in swimmers occurred during dynamic humeral elevation, which may be explained by the symmetrical nature of swimming (McKenna, Straker et al. 2011). For sport and clinical assessments, no bilateral differences in scapular upward rotation during dynamic humeral elevation up to $120^{\circ}$ in the scapular plan may be a significant outcome for shoulder 
injury prevention (McLaine, Ginn et al. In Press). Contrary to our initial hypothesis, scapular tilt was not altered by swimming exposure. The absence of adaptation in scapular anterior/posterior tilt may be explained by the intra-group variability, especially in our control group, and/or by the error of measurement in relation with measurements using electromagnetic system (Karduna, McClure et al. 2001). Concerning the last degree of freedom of the scapula, only the adult elite swimmers presented increased scapular internal rotation, especially at humeral elevation near $120^{\circ}$, suggesting that such adaptation would be generated by the accumulation of years of swimming practice at high level. Consequently, swimming may have differential effect on adaptations in scapular kinematics.

Our study displays some limitations that warrant discussion. A first limitation concerns the lack of a group composed of club-level adolescent swimmers, which would allow the assessment of the interaction effect of age and skill-level. Therefore, it was assumed that comparison between adult club-level (G3_AduClub) and adult elite (G2_AduElit) groups revealed the effect of skilllevel, while the comparison between adolescent (G1_AdoElit) and adult elite (G2_AduElit) groups gave the effect of age. A second limitation was that only men were enrolled in this study. Therefore, future studies with larger sample should also focus on women to assess a possible sex effect on scapular adaptations in swimmers. A third limitation was that the data was not collected in water, yet different adaptations could have been observed in such environment. Nevertheless, no current instrumentation allows accurate measurements of the scapula in water condition (Pink, Perry et al. 1991). Despite these limitations, the present study is the first to assess the shoulder and scapular kinematics throughout the arm elevation in the scapular plane to address the shoulder sport-specific adaptations in swimmers. It could be useful to include scapular kinematics measurements in a follow-up study to better understand how such scapular adaptations occur during the swimmer's career. Further studies also need to determine the influence of preferred swimming style on the shoulder and scapular kinematics, as well as to 
investigate the shoulder and scapular kinematics during dynamic humeral elevation in swimmers with symptomatic shoulders in order to understand the mechanisms leading to shoulder pathology.

\section{Conclusion}

This study confirmed that, in the context of humeral motion up to $120^{\circ}$ in the scapular plane, swimmers presented forward posture evidenced by clavicular protraction. Our results also pointed out that bilateral differences in scapular upward rotation were eliminated in swimmers, suggesting safe adaptations of their non-dominant shoulder. In addition, the adaptations in scapular internal rotation may be due to the accumulation of years of practice at high level. Finally, the findings of this study provide new knowledge on the adaptations in shoulder and scapular kinematics in relation with the age and level of swimming. In particular, focusing on clavicular retraction, scapular external rotation and bilateral symmetry in scapular upward rotation during humeral elevation may be objectives for swimming coaches and physicians to better prevent and rehabilitate shoulder injury in swimmers. Nevertheless, further studies are needed to assess the relationship between scapular kinematics and shoulder injuries in swimmers of different ages, style preference and levels.

\section{Acknowledgments}

none

\section{References}

Bak, K. (2010). "The practical management of swimmer's painful shoulder: etiology, diagnosis, and treatment." Clin J Sport Med 20(5): 386-390. 
Bak, K. and S. P. Magnusson (1997). "Shoulder strength and range of motion in symptomatic and pain-free elite swimmers." Am J Sports Med 25(4): 454-459.

Bradley, J., D. Bowmaker and J.-F. Gomez (2016). "Review of shoulder injuries and shoulder problems in competitive swimmers." American Journal of Sports Science and Medicine 4(3): 57-73.

Chollet, D., S. Chalies and J. C. Chatard (2000). "A new index of coordination for the crawl: description and usefulness." Int J Sports Med 21(1): 54-59.

$\mathrm{Du}, \mathrm{T}$. and T. Yanai (2016). Three dimensional motion of shoulder complex during front crawl swimming. 34rd International Conference in Biomechanics in Sports, Tsukuba, Japan.

El Habachi, A., S. Duprey, L. Cheze and R. Dumas (2015). "A parallel mechanism of the shoulder—application to multi-body optimisation." Multibody Syst Dyn 33(4): 439445.

Forthomme, B., J. M. Crielaard and J. L. Croisier (2008). "Scapular positioning in athlete's shoulder : particularities, clinical measurements and implications." Sports Med 38(5): 369-386.

Gaunt, T. and N. Maffulli (2012). "Soothing suffering swimmers: a systematic review of the epidemiology, diagnosis, treatment and rehabilitation of musculoskeletal injuries in competitive swimmers." Br Med Bull 103(1): 45-88.

Gillet, B., C. Berger-Vachon and I. Rogowski (2015). "Scapulothoracic kinematics during scaption after one year of tennis practice in elite girl players." Comput Methods Biomech Biomed Engin 18 Suppl 1: 1946-1947.

Gordon, R. (2004). "A shorter guide to long term athlete development (LTAD)." from http://www.soundwellswim.org.uk/. 
Hibberd, E. E., K. Laudner, D. J. Berkoff, K. L. Kucera, B. Yu and J. B. Myers (2016). "Comparison of Upper Extremity Physical Characteristics Between Adolescent Competitive Swimmers and Nonoverhead Athletes." J Athl Train 51(1): 65-69.

Hibberd, E. E., S. Oyama, J. T. Spang, W. Prentice and J. B. Myers (2012). "Effect of a 6-week strengthening program on shoulder and scapular-stabilizer strength and scapular kinematics in division I collegiate swimmers." J Sport Rehabil 21(3): 253-265.

Karduna, A. R., P. W. McClure, L. A. Michener and B. Sennett (2001). "Dynamic measurements of three-dimensional scapular kinematics: a validation study." $\underline{\mathbf{J}}$ Biomech Eng 123(2): 184-190.

Kebaetse, M., P. McClure and N. A. Pratt (1999). "Thoracic position effect on shoulder range of motion, strength, and three-dimensional scapular kinematics." Arch Phys Med Rehabil 80(8): 945-950.

Kibler, W. B., P. M. Ludewig, P. W. McClure, L. A. Michener, K. Bak and A. D. Sciascia (2013). "Clinical implications of scapular dyskinesis in shoulder injury: the 2013 consensus statement from the 'Scapular Summit'." Br J Sports Med 47(14): 877-885.

Laudner, K. G., M. Wenig, N. M. Selkow, J. Williams and E. Post (2015). "Forward Shoulder Posture in Collegiate Swimmers: A Comparative Analysis of Muscle-Energy Techniques." J Athl Train 50(11): 1133-1139.

Ludewig, P. M. and T. M. Cook (2000). "Alterations in shoulder kinematics and associated muscle activity in people with symptoms of shoulder impingement." Phys Ther 80(3): 276-291.

Ludewig, P. M., V. Phadke, J. P. Braman, D. R. Hassett, C. J. Cieminski and R. F. LaPrade (2009). "Motion of the shoulder complex during multiplanar humeral elevation." $\underline{\mathrm{J}}$ Bone Joint Surg Am 91(2): 378-389. 
Madsen, P. H., K. Bak, S. Jensen and U. Welter (2011). "Training induces scapular dyskinesis in pain-free competitive swimmers: a reliability and observational study." Clin J Sport Med 21(2): 109-113.

Matsuki, K., Matsuki, K.O., Mu, S., Yamaguchi, S., Ochiai, N., Sasho, T., Sugaya, H., Toyone, T., Wada, Y., Takahashi, K., Banks, S.A. (2011). "In vivo 3-dimensional analysis of scapular kinematics: comparison of dominant and nondominant shoulders." Journal of Shoulder and Elbow Surgery 20: 659-665.

McClure, P. W., L. A. Michener, B. J. Sennett and A. R. Karduna (2001). "Direct 3-dimensional measurement of scapular kinematics during dynamic movements in vivo." J Shoulder Elbow Surg 10(3): 269-277.

McKenna, L., L. Straker, A. Smith and J. Cunningham (2011). "Differences in scapular and humeral head position between swimmers and non-swimmers." $\underline{\text { Scand J Med Sci }}$ Sports 21(2): 206-214.

McLaine, S. J., K. A. Ginn, J. W. Fell and M.-L. Bird (In Press). "Scapular upward rotation position is symmetrical in swimmers without current shoulder pain." Phys Ther Sport.

Morais, N. V., Pascoal, A.G. (2013). "Scapular positioning assessment: Is side-to-side comparison clinically acceptable?" Manual Therapy 18: 46-53.

Myers, J. B., K. G. Laudner, M. R. Pasquale, J. P. Bradley and S. M. Lephart (2005). "Scapular position and orientation in throwing athletes." Am J Sports Med 33(2): 263-271.

Pataky, T. C. (2008). "Generalized n-dimensional biomechanical field analysis using statistical parametric mapping." Journal of Biomechanics 43: 1976-1982.

Pink, M., J. Perry, A. Browne, M. L. Scovazzo and J. Kerrigan (1991). "The normal shoulder during freestyle swimming. An electromyographic and cinematographic analysis of twelve muscles." Am J Sports Med 19(6): 569-576. 
Sein, M. L., J. Walton, J. Linklater, R. Appleyard, B. Kirkbride, D. Kuah and G. A. Murrell (2010). "Shoulder pain in elite swimmers: primarily due to swim-volume-induced supraspinatus tendinopathy." Br J Sports Med 44(2): 105-113.

Seitz, A. L., R. I. McClelland, W. J. Jones, R. A. Jean and J. R. Kardouni (2015). "A Comparison of Change in 3d Scapular Kinematics with Maximal Contractions and Force Production with Scapular Muscle Tests between Asymptomatic Overhead Athletes with and without Scapular Dyskinesis." Int J Sports Phys Ther 10(3): 309318.

Senk, M. and L. Chèze (2010). "A new method for motion capture of the scapula using an optoelectronic tracking device: a feasibility study." Comput Methods Biomech Biomed Engin 13(3): 397-401.

Van de Velde, A., K. De Mey, A. Maenhout, P. Calders and A. M. Cools (2011). "Scapularmuscle performance: two training programs in adolescent swimmers." $\underline{\mathrm{J} \text { Athl Train }}$ 46(2): 160-169.

Wu, G., F. C. van der Helm, H. E. Veeger, M. Makhsous, P. Van Roy, C. Anglin, J. Nagels, A. R. Karduna, K. McQuade, X. Wang, F. W. Werner, B. Buchholz and B. International Society of (2005). "ISB recommendation on definitions of joint coordinate systems of various joints for the reporting of human joint motion--Part II: shoulder, elbow, wrist and hand." J Biomech 38(5): 981-992.

Yanai, T. and J. G. Hay (2000). "Shoulder impingement in front-crawl swimming: II. Analysis of stroking technique." Med Sci Sports Exerc 32(1): 30-40. 